\title{
Acoustic comfort in the living environment and its association with noise representation: A systematic review
}

\author{
Farrah Jahangeer ${ }^{1, *}$ \\ ${ }^{1}$ Ecole Nationale Supérieure d'Architecture de Nantes - Mauritius
}

\begin{abstract}
Noise is commonly known to have adverse health effects on residents in urban living environment. In the past, great focus has been placed on the impact of external traffic noise on residents' health and the development of urban noise mapping. On the other hand, neighbourhood noise - a combination of noise generated from outside residences and noise generated from within residences - are also considered to be as detrimental as external traffic noise and are yet to be widely investigated. This paper focuses on the systematic review of studies assessing neighbourhood noise with the intention of determining the presence of architectural variables and methods of noise representation for micro-scale areas. 153 articles are retrieved from specific research databases and out of which 12 were considered to be relevant to the study. The results indicate that architectural variables present in the studies were mostly related to the analysis of external traffic noise and that representation of noise in analyses were rarely devised which highlights the need for further investigation in this area.
\end{abstract}

\section{Introduction}

This paper is a review of studies assessing noise annoyance in residences with the intention of determining the association with representation of both architectural parameters and the aural environment. The rise in global urban population generates greater need for economically designed multi-storey houses providing sufficient comfort to residents. The increasing tendency in designing and building micro-houses [1] and multi-storeys further increases housing density which questions the intensity and impact of structure-borne and airborne-noise experienced by residents.

Over the decades, the impact of traffic noise on residents' health have been widely investigated and published. For instance, Belojevic et al. (1997) observed in a comparative study that residents in noisy traffic areas $\left(72 \mathrm{dBA}_{\text {leq }}\right)$ experienced more fatigue, headaches, nervousness and sleep disturbances in comparison to other residents exposed to less urban noise $\left(56 \mathrm{dBA}_{\text {Leq }}\right)$. A meta-analysis study showed that residents with constant noise exposure to traffic noise above $55 \mathrm{dBA}$ had high risks of cardiovascular disease [2]. However, noise

\footnotetext{
*Corresponding author: farrah.jahangeer@nantes.archi
} 
experienced by residents, also known as 'neighbour noise' or 'neighbourhood noise', are caused by multiple sources linked to neighbour activities (TV, music, voices, footsteps and so on), airborne noise from outside (traffic) and noise from building services. Neighbour noise could be considered a result of dynamic events occurring in residences and the perception of this type of noise is likely to be subjective to culture and type of built environment.

The literature in the field of room acoustics, psychoacoustics and built environment has very few rigorous studies assessing noise dissatisfaction in dwellings. In a recent review evaluating the relationship between acoustic field data and subjective responses in dwellings, Vardaxis et al. [3] found only 10 complete studies since 1985 . While noise in residences may not be as critical as noise in the workplace determining work performance, associations between neighbour noise and health have been observed in several studies; Maschke \& Niemann [19] highlighted that annoyance with neighbour noise was related to stress and increased health risks in cardio-vascular systems in a cross-sectional study across Europe; Rautio et al. [4] observed that noise (from airborne or structural) experienced by residents was significantly associated with depressive mood of residents.

In the field of room acoustics, physical aspects of rooms or spaces are important measures to be considered. Sabine's equation [5] for reverberation time established since the $20^{\text {th }}$ century demonstrates the link between volume of room and absorptive surface area. In a laboratory experiment investigating the perception of distance of noise source in relation to reverberation time, Mershon et al. [6] reported that in spaces with longer reverberation time (1.36s) subjects tended to overestimate the distance of noise sources than those in settings with shorter reverberation time $(0.36 \mathrm{~s})$. Similar results were reported by Cabrera \& Gilfillan [7] where perceived distance of speech source was found to increase with reverberation time. Mommertz [8] also indicates the relationship between the shape of rooms and variations in sound propagation in the related space. The aural environment, is described as sounds being integrated in a space or built environment that contributes to the experiential identity of the space [9] and the graphic two-dimensional representation of such environment is yet to be sought. Despite the advances in auralization to virtually experience three dimension spaces, architectural designs at concept stages commence with two-dimensional sketches, which accentuates the ability to represent the aural environment in two dimensions. Alternatively, graphic noise representation on a large scale in the urban environment, known as 'noise mapping' is greatly being investigated in the recent years with the support of developing technology and programs thereby assisting in the monitoring and control of noise pollution.

This review evaluates past studies assessing noise dissatisfaction in residences with the aim of investigating the following research question: to what extent is architecture and the aural environment being represented when analysing neighbour noise? The following sections describes the methodology applied in selecting articles for the review and their evaluation.

\section{Review methodology}

A similar systematic review to that used in the study of Rautio et al. [4] was used here including three specific stages in the review. Articles used in this study were populationbased studies with peer-reviews published in academic journals. In the first stage, the following journal databases - Sciencedirect, Sage, BIB CNRS and JSTOR - were researched manually for articles with titles including one of the three keywords to ensure that all articles addressing noise issues in the living environment were included: 'domestic noise', 
'residential noise' and 'neighbour noise'. These three different keywords relate to different degrees of living proximities and the focus in this study is on the evaluation of neighbour noise experienced by indoor residents. In the second stage, articles assessing neighbour noise with objective and subjective measures were selected and their abstracts read. Acoustic studies focusing on aircraft and railway noise were excluded from the study because these specific noise sources were considered to be unrepresentative of typical central urban residential areas in cosmopolitan cities. In the third stage, the selected articles were fully read and the quality of the articles was rated based on the following inclusion of the following variables: architectural parameters, urban setting variables, subjective noise rating, objective acoustic measures, graphic noise representation and significant statistical results. One point was attributed to the presence of the aforementioned variables.

\section{Results}

\subsection{Study selection}

At the first stage, the keyword search in the aforementioned databases led to the retrieval of 153 articles- domestic noise: 33 , residential noise: 86 , neighbour noise: 34 . The selected studies at that stage in relation to the time of publication, as shown on the graph in Figure 1, indicates the recent increasing awareness and concern with noise in the living environment. Studies focusing on 'residential noise' investigated mainly noise caused by vehicles and its impact on health and economy. Studies focusing on 'neighbour noise' analysed mainly the transmission of noise caused by neighbour activities in relation to construction systems and sound insulating materials. Studies investigating 'domestic noise' focused mainly on isolated noise sources, such as pets and air conditioning units. After the second stage of the selection process, only 12 complete studies met the above selection criteria and these were evaluated in the final stage.

\subsection{Study characteristics}

As seen in the Table 1, 6 studies were carried out in Europe, 6 in East Asia and 1 in USA. Most of the studies had cross-sectional research designs and only 3 with experimental laboratory research designs indicating the prevalence for population based survey at a single point in time which is most likely linked to the restricted access to dwellings. In all studies in this review, subjective ratings of annoyance with neighbour noise were recorded for varying neighbour noise sources. Acoustic measures present in some of the studies here were weighted sound levels (dBA) and sound insulation index. Architectural parameters such as spatial measurements were not present but other variables such as dwelling typology and perceived distances were present. Sample sizes in the review varied between 20 and 9000. Age of sample populations in most studies varied between 18 and 90 years. In some singular cases, Wang et al.[10] only considered the age group of 20 to 26 years because of the expected decline in hearing acuity. Both males and females were considered in all studies but Jeon et al. [11] highlighted that its sample contained mainly (73\%) housewives. 


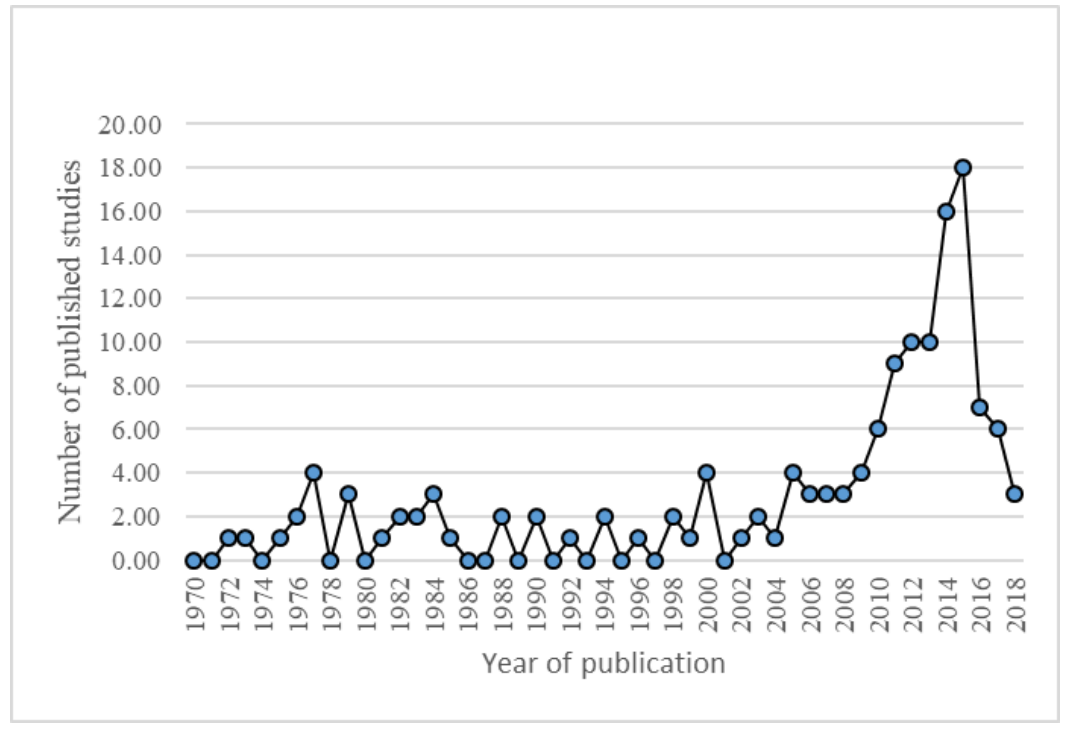

Figure 1. Number of studies published over the years after searching the databases for the specific keywords 


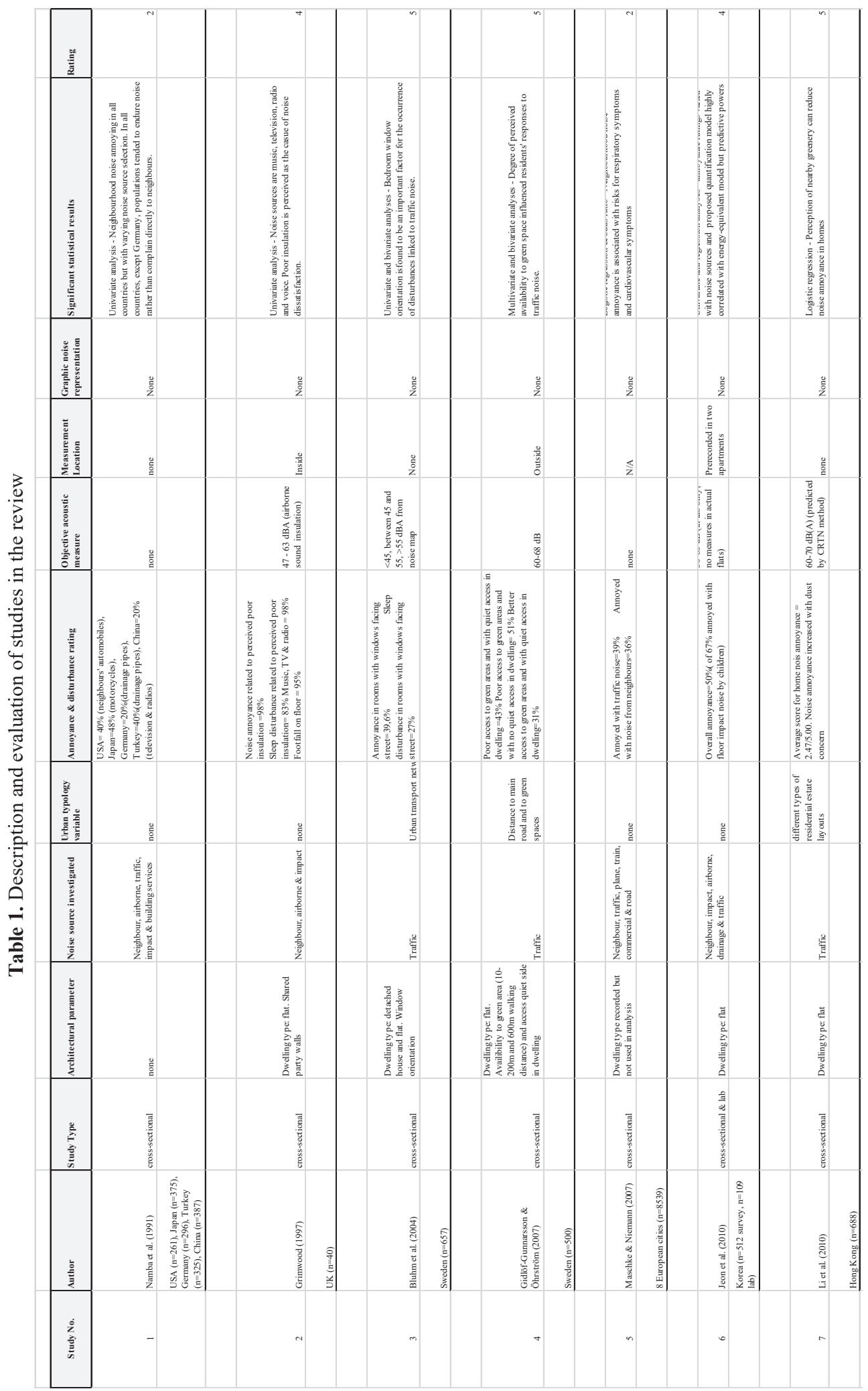




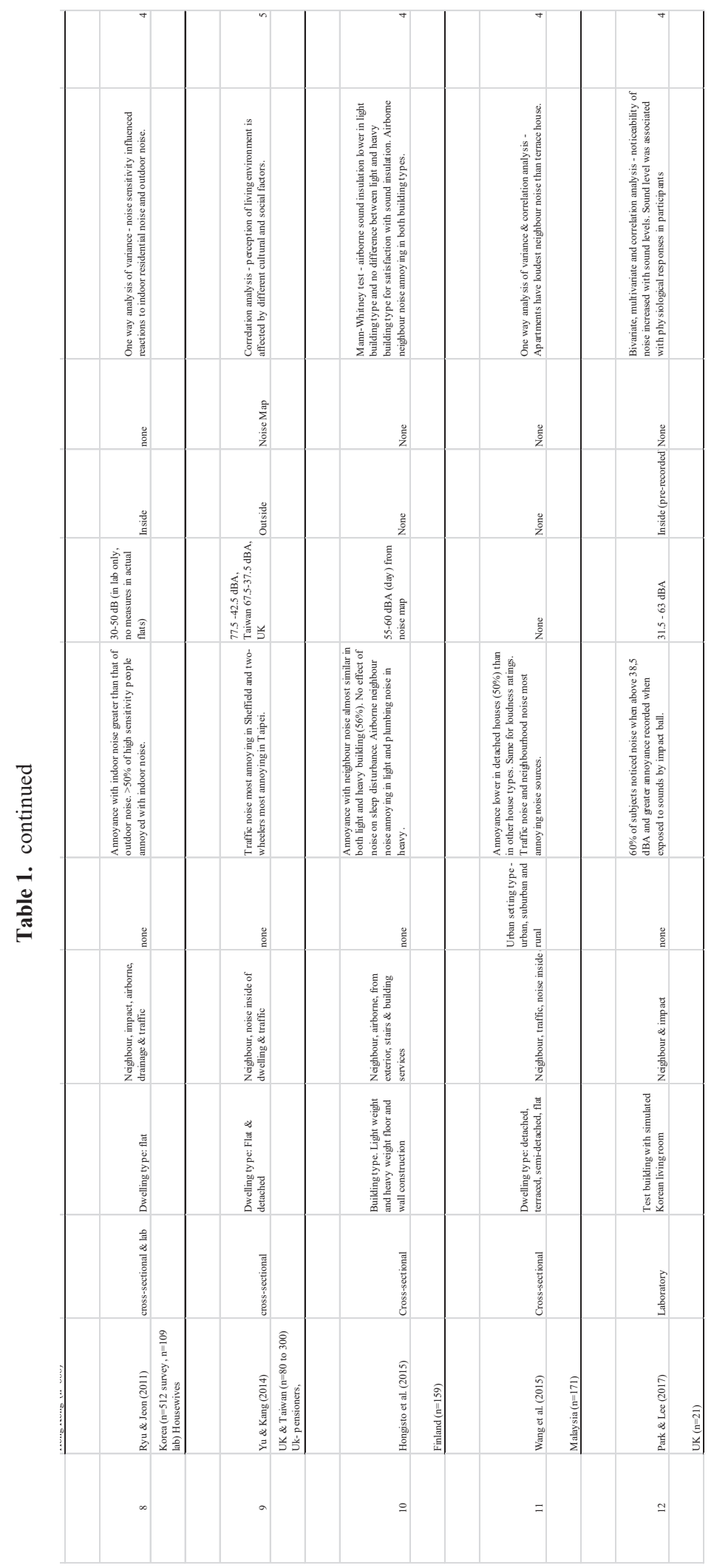




\subsection{Objective acoustic measures}

Noise sources being investigated tended to vary; airborne noise, impact noise, traffic noise, building services noise and other external types of noises (such as commercial noise). Noise levels of sources were considered in only nine studies and were determined by various methods. Study 3,4 and 7 in Table 1 focusing mainly on traffic noise had noise levels varying between $43 \mathrm{dBA}$ and $70 \mathrm{dBA}$. Bluhm et al. [12] derived traffic noise levels from noise maps available while Gidlöf-Gunnarsson \& Öhrström [13] and Li et al. [22] derived noise levels from CRTN (calculation of traffic road noise) method. Gidlöf-Gunnarsson \& Öhrström [13] further referred to the speed of the vehicles which according to UK Noise Association [14] a reduction in $10 \mathrm{~km} / \mathrm{h}$ can reduce noise level by $40 \%$. Other parameters considered to influence traffic noise levels, such as speed zones of areas being investigated, road surface textures and types of vehicles in the investigated areas were not mentioned. Hongisto et al. [15], on the other hand, determined the building selection for their study according to noise maps highlighting areas with traffic noise levels between 50 to $60 \mathrm{dBA}$.

Other six studies (Study 2, 6, 8, 9, $10 \& 12$ ) investigated several noise sources and the range of noise levels varied considerably, between $30 \mathrm{dBA}$ and $63 \mathrm{dBA}$. In the laboratory experiment by Jeon et al. [11] annoyance was evaluated in relation to each noise source separately. None of the studies included noise levels recorded within dwellings for a defined period to fully represent the soundscape in dwellings. Grimwood [16] and Hongisto et al. [15] measured the sound reduction index and sound insulation in relation to noise transmission through walls and floors. Reverberation time, the acoustic measure used to define quality of acoustics, was not used in the studies reviewed here.

\subsection{Representation of acoustic measures}

The requirements of the European Commission to produce noise maps every 5 years for specific areas by member states have further encouraged the use of noise maps in research (END 2002). A few studies in the review (Study 3, $9 \& 10$ ) have used noise maps to derive estimates of noise levels of the areas being investigated in relation to the built area and density (see Figure 2). The noise maps used were similar to those produced by Scotland's Environment and accessible by the public (see Figure 2). The noise maps are required to be updated every five years according to the Environmental Noise Directive. No other forms of two-dimensional graphic representation of noise, such as location points of noise sources in dwellings, were used in the studies to represent the aural environment and its variation with time.

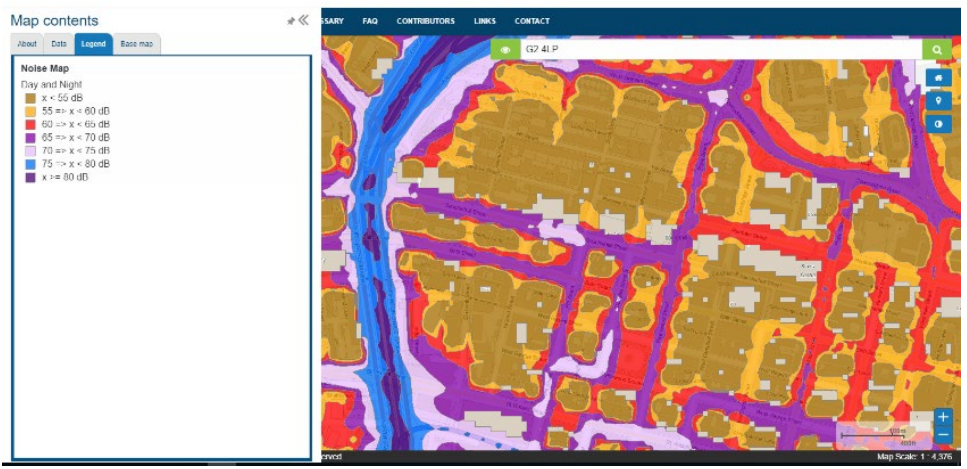

Figure 2. Noise maps available on Scotland's Environment. 


\subsection{Architectural parameters}

The review indicates that a few architectural parameters were used in the analyses of noise annoyance in living environments, such as housing typologies, spatial urban context, construction type and orientation of windows in dwellings. Housing typologies were used in the analysis of studies 3, 9 and 11. Bluhm et al. [12] analysed both detached houses and flats and the window orientation in both dwelling type suggesting that annoyance with traffic noise was $16.3 \%$ less in dwellings with windows facing gardens than those facing the street. However, there was no mention of glazing ratio or technical details of windows. Yu \& Kang [17] considered two types of housing; detached houses and flats but failed to compare the subjective data collected between the two. Wang et al. [10] performed a comparative analysis for subjective data collected in four types of dwellings; detached, terraced, semi-detached and flat and indicating that perceived noise annoyance was highest in flats.

Gidlöf-Gunnarsson \& Öhrström [13] observed that flats with one side having average noise level less than 48dBA and located within walking distances to green areas $(10 \mathrm{~m}$ to $200 \mathrm{~m}$ ) had $20 \%$ less annoyance than flats with higher average noise level and with further walking distances to green areas. Hongisto et al. [15] investigated the relationship between building construction type -light and heavy weight - in relation to neighbour noise transmission and specified the construction period and the materials used for walls and floors. Light weight floor materials consisted of parquet, soft underlay, cast concrete, flexible isolation board, steel reinforced concrete and walls consisted of plasterboard, mineral wool steel studs and plasterboard. Heavy weight floors and walls consisted of steel reinforced concrete. This study also mentioned the number of floors in the buildings being surveyed. Park \& Lee [18] referred to the simulation of living rooms in the laboratory experiment providing details of surface material finishes and construction materials used - volume of $38 \mathrm{~m}^{3}$, wooden flooring and furniture and concrete structure. However, there were no further details on the layout of the space, openings or location of participants during study. Similarly, no other architectural elements were present in other studies reviewed here such as the floor plan of dwellings and architectural parameters that have been analysed so far could be considered sparse and superficial.

While urban context also contributes to the aural environment in which dwellings are located only few studies have considered parameters in the urban environment. Bluhm et al. [12] considered the type of urban network surrounding the residences and Gidlöf-Gunnarsson \& Öhrström [13] analysed the distance between green spaces and buildings being surveyed. Li et al. [22] and Wang et al. [10] investigated the type of residential setting (e.g. urban, suburban or rural). There were no other mentions of urban form, urban pattern, building intensity or building heights which can be expected to influence noise propagation.

\subsection{Subjective satisfaction measures}

All of the studies in the review assessed perceived noise annoyance but the types of noise sources being evaluated differed. To begin with two different definitions of annoyance were observed in the review; Jeon et al. [11] defined annoyance as a feeling of displeasure associated with any agent or condition while Maschke \& Niemann [19] defined annoyance as a feeling of discomfort which is related to adverse influence on an individual by any substance or circumstance. Traffic noise and noise arising from neighbour activities (such as TV, music, talking) were the most investigated noise sources in the review. Table 2 indicates the percentage rating reported in the studies here except of three studies (Study $4,9 \& 12$ ) that were excluded because of the type of research study (laboratory based) and 
missing data. Based on the average noise annoyance ratings available in the studies, neighbour noise was found to have higher noise ratings than annoyance caused by traffic noise which highlights the need for further investigation into neighbour noise. Some studies, such as that of Maschke \& Niemann [19], evaluated neighbour noise as one cluster while in other studies, such as that of Jeon et al. [11] each noise source was evaluated separately. This distinction of noise sources is likely to vary according to households and culture. For instance, in the study of Namba et al. [20] drainage sounds were found to be the most annoying in West Germany and Turkey whereas in China sound from television and radios were the most annoying. In Sheffield, traffic noise was rated as the most annoying noise source whereas in Taipei talking, music and TV were the most annoying noise sources $(\mathrm{Yu}$ \& Kang 2014).

Table 2. Percentage ratings reported in studies reviewed here.

\begin{tabular}{|c|c|c|c|c|c|c|c|}
\hline & \multicolumn{7}{|c|}{ Average percentage of annoyance ratings } \\
\hline Study no. & traffic & voices & music & TV & $\begin{array}{l}\text { impact } \\
\text { noise }\end{array}$ & drainage & stairs/circulation \\
\hline 1 & - & 16.6 & 20.2 & 14.2 & & & 18.6 \\
\hline 2 & - & 78 & \multicolumn{2}{|c|}{98} & 95 & 50 & - \\
\hline 3 & 12.8 & - & _ & - & _ & - & - \\
\hline 5 & 39 & \multicolumn{4}{|c|}{36} & _- & - \\
\hline 6 & 87 & \multicolumn{2}{|c|}{65} & - & 67 & 60 & - \\
\hline 7 & 49.4 & - & - & - & - & - & - \\
\hline 8 & 87 & \multicolumn{4}{|c|}{67} & 60 & - \\
\hline 10 & 42 & \multicolumn{3}{|c|}{43} & & & \\
\hline 11 & 49.1 & \multicolumn{3}{|c|}{57.5} & _ & _ & _ \\
\hline
\end{tabular}

Other independent variables present in dwellings that could possibly be linked with noise annoyance were also evaluated; Hongisto et al. [15] assessed noise sensitivity and extraversion of participants, $\mathrm{Li}$ et al. [22] evaluated the relationship between perceived foul odour from exterior and perceived annoyance with traffic noise, Gidlöf-Gunnarsson \& Öhrström [13] assessed subjective psychosocial stress in relation to traffic noise annoyance and $\mathrm{Yu} \&$ Kang [17] analysed the correlation between perception of quiet living environment and education level and age groups.

The methodology deployed for collection of subjective data varied considerably. Questionnaires were mostly administered in two ways - by post or verbally during interviews. The response rate was reported in several studies (Study 3,4,7,10 \& 11) and was above $50 \%$ except for study number 11 where the response rate was $17.1 \%$. The studies are in line with the recommended response rate between $40 \%$ and $80 \%$ as suggested by Baruch [21]. The evaluation scales also varied - 5-points scale, 7-points scale, 10-points scale and several studies such as that of Gidlöf-Gunnarsson \& Öhrström [13] and Li et al. [22] used different scales thereby providing the possibility of counterchecking responses obtained. While correlation coefficients are indicative of the type of association present in the aforementioned studies, the causality is difficult to predict in such types of research designs.

\subsection{Study evaluation}

After evaluation of each study, the highest score achieved was 5 and with an average of 4 . The majority of the studies scoring 4 highlighted the absence of acoustic measures and urban 
typology variables in the studies. The lowest score obtained was 2 in the study of Maschke \& Niemann [19] where only subjective data was collected in a large cross-sectional study across Europe. Four cross-sectional studies scored 5 and out of these three focused entirely on annoyance with traffic noise and included either measured noise levels or noise levels estimated from noise maps.

\section{Discussion}

The review of studies evaluating noise annoyance in the living environment brings to light the complexities associated with such studies and the areas that could be further explored. The definition of 'neighbour noise' needs further precision because clusters of different noise sources in dwellings were given similar name - Maschke \& Niemann [19] considered neighbour noise as the presence of voices, TV, music and impact noise while Jeon et al. [11] considered only voices and music as neighbour noise. The demography of studies does not indicate significant findings when assessing age but gender and occupation seem to be important factors given that in some studies housewives were main participants in surveys. The aural experience of housewives is likely to differ from those of their husbands and same goes for employees working from homes.

In general, great importance is given to the destructive impact of traffic noise on health and annoyance - WHO [23] recommends an average night exposure of $40 \mathrm{dBA}$ away from busy streets. However, the findings here indicate greater annoyance with neighbour noise which is most likely because of its unpredictable and uncontrollable characteristics. Noise levels provide some indication of intensity of noise but does not necessarily reflect noise annoyance of inhabitants. Guski [24] reported that annoyance of inhabitants were only $33 \%$ dependent on acoustic measures and therefore other measures are required to fully characterize the aural environment of dwellings. It is considered that acoustics measures and architectural spatial parameters investigated in the studies were sporadic and do not fully describe the aural environment of dwellings Furthermore, the aural environment is likely to vary in different cultures and the relationship between cultural geographies of home [25] and noise perception is yet to be established. The difficulty of accessing dwellings for long periods of time could be considered as a limiting factor in studies here.

The Environmental Noise Directive in 2002 initiatives triggered the increased use of noise maps but these have certain shortcomings when assessing noise on microscales and temporality of noise. Alternative noise mapping strategies, such a smartphone-based noise mapping could be sought to obtain more frequent and accurate data whilst developing a participatory approach in field surveys. The two-dimensional graphic representation of aural environment is an essential element that could be integrated in early stages of architectural designs and studies here have not shown any association between any form of representation of the aural environment and noise annoyance in dwellings.

\section{Conclusion}

The review here assessed studies investigating noise annoyance in living environments and the findings indicate that the integration of architectural measures and the representation of architecture and the aural environment are sparse. Further investigation is required to fully comprehend the transient nature of aural environments in dwellings. There are insufficient data, be it subjective or objective, on neighbour noise and more investigations are required in the field to improve acoustic comfort in dwellings. The limitation of data collection caused 
by access difficulties in dwellings require the development of innovative methodologies and measures for data collection. The evaluation of subjective acoustic measures occurs in several countries and some even take into account the influence of culture when assessing noise perception in cross-sectional studies. The association between spatial organisation of dwellings across cultures and noise annoyance ratings could be further possible areas of investigations.

\section{References}

1. R. Post, "Are tiny houses and micro-apartments the future of urban homes? | Guardian Sustainable Business | The Guardian," 2014.

2. T. Münzel, T. Gori, W. Babisch, and M. Basner, "Cardiovascular effects of environmental noise exposure.," Eur. Heart J., vol. 35, no. 13, pp. 829-36, Apr. 2014.

3. N.-G. Vardaxis, D. Bard, and K. Persson Waye, "Review of acoustic comfort evaluation in dwellings - part I: Associations of acoustic field data to subjective responses from building surveys," Build. Acoust., vol. 25, no. 2, pp. 151-170, Jun. 2018.

4. N. Rautio, S. Filatova, H. Lehtiniemi, and J. Miettunen, "Living environment and its relationship to depressive mood: A systematic review," Int. J. Soc. Psychiatry, vol. 64, no. 1, pp. 92-103, Feb. 2018.

5. W. Furrer, Room and Building Acoustics and Noise Abatement. London: Butterworths, 1964.

6. D. Mershon, W. Ballenger, A. Little, P. McMurty, and J. Buchanan, "Effects of room reflectance and background noise on perceived auditory distance," Perception, vol. 18, pp. 403-416, 1989.

7. D. Cabrera and D. Gilfillan, "Auditory distance perception of speech in the presence of noise," in Proceedings of the 2002 International Conference on Auditory Display, Kyoto, Japan, 2002.

8. E. Mommertz, Acoustics and sound insulation: principles, planning, examples. Basel: Birkhäuser, 2009.

9. T. Sheridan, N. York, and K. Van Lengen, "Hearing Architecture Exploring and Designing the Aural Environment,” ACSA, Inc, 2003.

10. C. Wang, Y. Si, H. Abdul-Rahman, and L. C. Wood, "Noise annoyance and loudness: Acoustic performance of residential buildings in tropics," Build. Serv. Eng. Res. Technol., vol. 36, no. 6, pp. 680-700, Nov. 2015.

11. J. Y. Jeon, J. K. Ryu, and P. J. Lee, “A quantification model of overall dissatisfaction with indoor noise environment in residential buildings," Appl. Acoust., vol. 71, no. 10, pp. 914-921, Oct. 2010.

12. G. Bluhm, E. Nordling, and N. Berglind, "Road traffic noise and annoyance--an increasing environmental health problem.," Noise Health, vol. 6, no. 24, pp. 43-9, 2004.

13. A. Gidlöf-Gunnarsson and E. Öhrström, "Noise and well-being in urban residential environments: The potential role of perceived availability to nearby green areas," Landsc. Urban Plan., vol. 83, no. 2-3, pp. 115-126, Nov. 2007. 
14. P. Mitchell, "Speed and Road Traffic Noise The role that lower speeds could play in cutting noise from traffic A report commissioned by the UK Noise Association. Researched and written by Paige Mitchell," 2009.

15. V. Hongisto, M. Mäkilä, and M. Suokas, "Satisfaction with sound insulation in residential dwellings - The effect of wall construction," Build. Environ., vol. 85, pp. 309-320, Feb. 2015.

16. C. Grimwood, "Complaints about poor sound insulation between dwellings in england and wales," Appl. Acoust., vol. 52, no. 3-4, pp. 211-223, Nov. 1997.

17. C.-J. Yu and J. Kang, "Soundscape in the sustainable living environment: A crosscultural comparison between the UK and Taiwan," Sci. Total Environ., vol. 482483, pp. 501-509, Jun. 2014.

18. S. H. Park and P. J. Lee, "Effects of floor impact noise on psychophysiological responses," Build. Environ., vol. 116, pp. 173-181, May 2017.

19. C. Maschke and H. Niemann, "Health effects of annoyance induced by neighbour noise," Noise Control Eng. J., vol. 55, no. 3, p. 348, 2007.

20. S. Namba, S. Kuwano, A. Schick, A. Aclar, and M. Florentine, "A cross-cultural study on noise problems: comparison of the results obtained in Japan, West Germany, The U.S.A., China and Turkey," J. Sound Vib., vol. 151, no. 3, pp. 471477, 1991.

21. Y. Baruch, "Response Rate in Academic Studies - A Comparative Analysis," Hum. Relations, vol. 52, no. 4, pp. 421-438, 1999.

22. H. N. Li, C. K. Chau, and S. K. Tang, "Can surrounding greenery reduce noise annoyance at home?," Sci. Total Environ., vol. 408, no. 20, pp. 4376-4384, Sep. 2010 .

23. "WHO night noise guidelines for Europe," Mar. 2019.

24. R. Guski, "Psychological determinants of train noise annoyance," Euro-Noise, vol. 98, no. 1, 1998.

25. A. Blunt, "Cultural geography: cultural geographies of home," Prog. Hum. Geogr., vol. 29, no. 4, pp. 505-515, Aug. 2005. 